\title{
El Proceso del Diseño Industrial como Herramienta de la Gestión de la Empresa
}

The Process of Industrial Design as a Tool for Business Management

\section{Arroyo F.1; Bravo. D.2}

${ }^{1}$ Universidad de Valladolid, Escuela de Ingenierías Industriales. Valladolid, España.

e-mail: flavio.arroyo@gmail.com

${ }^{2}$ Universidad Politécnica de Valencia, Escuela Técnica Superior de Ingeniería del Diseño.

Camí de Vera. Valencia, España.

e-mail: delydis@gmail.com

Información del artículo

Recibido: abril 2020

Aceptado: mayo 2020

\section{RESUMEN}

El diseño industrial es una valiosa herramienta que las empresas pueden implementar a su favor, lamentablemente pocas son las que están conscientes de que para el surgimiento de ellas, se debería desarrollar innovación a través del diseño tanto para productos como servicios, mediante la difusión de las tecnologías, el acortamiento del ciclo de vida de los productos y las estrategias empresariales que refieren a la necesidad de atender demandas, es decir, identificar oportunidades basados en modelos estratégicos los mismos que refuercen la competitividad de las empresas, sin olvidar la importancia de la innovación de productos, sistemas o servicios que manejen estrategias amigables con el medio ambiente, teniendo en cuenta la implementación de la economía circular, lo que favorece a las empresas en sus finanzas. Entonces la adaptación y modificación continua de sus productos a través de todos los medios mencionados anteriormente ayudarán a mantener su posición y obtener ventajas sobre sus competidores.

Palabras Clave: Diseño Industrial, Metodologías del Diseño Industrial, Gestión Estratégica, Innovación Empresarial, Modelos de Gestión.

\begin{abstract}
Industrial design is a valuable tool that companies can implement in their favour, unfortunately there are few who are aware that for the emergence of them, innovation should be developed through design for both products and services, by disseminating the Technologies, shortening the life cycle of products and business strategies that refer to the need to meet demands, that is, to identify opportunities based on strategic models that reinforce the competitiveness of companies, not forgetting the importance of innovation Of products, systems or services that handle environmentally friendly strategies, taking into account the implementation of the circular economy, which favors companies in their finances. Then the continuous adaptation and modification of your products through all means mentioned above will help to maintain your position or gain advantages over your competitors.
\end{abstract}

Keywords: Industrial Design, Industrial Design Methodologies, Strategic Management, Business Innovation, Management Models 


\section{Introducción}

El éxito de una empresa sin duda se debe a múltiples factores, que, al ser estructurados de forma correcta, resultan en herramientas claves para el renombre y posicionamiento de una entidad empresarial. Desde la década de los 70, estos factores han ido cambiando la forma de determinar la competitividad de las empresas. Consecuentemente el Diseño Industrial no puede existir sin empresas o industrias, las mismas que están vinculadas a un mercado y usuarios objetivos. Muchas de las veces las empresas omiten el diseño en sus actividades debido al incremento de gastos estancándose en el nulo desarrollo de patentes, competitividad mínima, mercados pequeños sin posibilidades de expansión y daños al medio ambiente.

Las empresas se han enfocado en producir y vender más barato y plasmar en sus productos cualidades y atributos que les permitan competir, reduciendo costos de producción que resultan ser una desventaja al compararlos con la competencia. Aquí radica la importancia el diseño, ya que gracias a su aporte se pueden generar productos y servicios que muestren un alto contenido simbólico sin olvidar lo estético funcional, para responder a las necesidades de los usuarios, además incrementa el valor económico de los productos al enmarcarlos en un contexto de calidad y buenos diseños.

Se define el diseño industrial como la actividad que prevé y define los requisitos de los productos y los proyecta tal y como se producirán posteriormente en serie [1]; es así como el diseño industrial constituye un recurso indispensable para el éxito de una empresa, repercutiendo directamente en la optimización de los recursos de la misma y en el éxito del producto en el mercado; sin embargo, su importancia aun no es entendida por muchas empresas. Según el artículo de [2] "Importancia del Diseño Industrial en la Gestión Estratégica de la empresa", se pone en manifiesto el valor que tiene el diseño en la empresa. Actualmente, el aumento de la competencia, globalización, mercados divididos, propagación de tecnologías, acortamiento del ciclo de vida de los productos y finalmente las nuevas exigencias que presentan los consumidores, muestra el aporte del diseño en la resolución de dichas necesidades, con el afán de mejorar la productividad de la empresa y estrechar lazos con los usuarios.
El diseño constituye un eje fundamental que permite promover el desarrollo de nuevos productos, funcionales y altamente eficientes, que satisfacen las necesidades propias del mercado y muestran el sello diferenciador que le otorga el valor agregado a la producción, motivo por el que las empresas que acogen al diseño como eje dentro de sus lineamientos, son capaces de diferenciarse, adaptarse e innovar sus productos, lo que les otorga una ventaja competitiva que les mantiene posicionados en el mercado.

El diseño participa en el posicionamiento estratégico y en el proceso de construcción de una ventaja competitiva [3]. El proceso de diseño es visto como una representación mental de un modelo de gestión que organiza de forma paralela la ingeniería y la innovación [4]. Por tal motivo abarca múltiples actividades, tales como investigación y desarrollo, las mismas que además de tener un carácter formal poseen un carácter creativo, que se mantienen en constante cambio, a fin de satisfacer las necesidades de los usuarios, sin olvidar la importancia de la innovación al fin de conseguir mejores diseños, reducción de costos y una calidad competente. Por tal motivo, la empresa que aplica el diseño adquiere una ventaja ante otras permitiéndole un mejor desempeño en el mundo globalizado.

En el presente artículo se definen los conceptos de mayor relevancia para el diseño industrial y la relación que este guarda con la gestión empresarial, además se analizará la importancia del diseño industrial como un instrumento del desarrollo de la gestión e innovación de lasempresas.

\section{Materiales y Método}

\subsection{Metodología}

Este trabajo emplea una metodología basada en la Investigación Científica y el Método Deductivo Indirecto para la posterior deducción de conclusiones a presentarse en este documento, las metodologías propuestas serán empleadas con el fin de identificar como el Diseño Industrial se integra a la Gestión Estratégica de una empresa constituyéndose como una herramienta. Para llegar a este objetivo se ha empezado con una fase previa, donde mediante la revisión bibliográfica de temas específicamente ordenados y vinculados al objetivo principal, se emiten importantes conclusiones y se 
plantea un posible modelo de gestión empresarial a partir de uno ya existente encontrado en la investigación.

El método deductivo indirecto emplea silogismos lógicos con lo que se compara premisas para ver la relación de están con otra, así se menciona en el documento [5].

Entre los elementos a investigar se resaltan: Metodologías del Diseño Industrial, Estratégica del Diseño, Gestión Estratégica Empresarial, Modelos de Estrategia Empresarial, Gestión Estratégica y el perfeccionamiento empresarial y la Innovación Empresarial, temas que están inmersos en el éxito empresarial con la inserción del Diseño Industrial.

\subsection{Diseño Industrial: definición, historia y conceptos}

El Consejo Internacional de Diseño Industrial (ICSID, International Council of Societies of Industrial Design) define el diseño como una actividad proyectual que se encarga de precisar las múltiples cualidades de objetos, procesos, servicios y sistemas en completos ciclos vitales; es por ello que el diseño es considerado como el eje central de la humanización innovadora de tecnologías y el factor crucial del intercambio económico y cultural. A esta definición se le puede agregar que el diseño industrial tiene como objetivo, resolver las complejas relaciones entre la forma y la función de los objetos capaces de ser producidos en serie, considerando los potenciales beneficios de la comercialización de estos objetos.

El diseño Industrial es una disciplina que se remonta a la revolución industrial (1750), pero es a principios de siglo XX donde toma forma como profesión y desde entonces se ha ido desarrollando paulatinamente. En esta época surgieron escuelas de arte, como, Arts and Crafts (artes y oficios) [6] y el Art Nouveau (Arte Nuevo), que participaron en el desarrollo del diseño de productos, con creaciones artesanales con altos contenido artístico. Pero, en 1919, la escuela de la Bauhaus (casa de construcción) [7], fundada en Alemania, fue la que propuso en su filosofa, la unión de la belleza y tradición de los oficios y las artes con las nuevas tecnologías industriales, en busca de que los productos, al ser más baratos y creados a mayor velocidad, puedan llegar a todos los estratos de lasociedad.
En los años sesenta el diseño industrial toma un mayor impulso, antes de esta época la demanda superaba ampliamente a la oferta de productos, pero esto se superó rápidamente gracias a la revolución de los transportes y comunicaciones, los cuales permitieron mayores intercambios comerciales y la economía empezó a globalizarse, lo que ocasionó una mayor competencia por el desarrollo empresarial y económico, la cual se ha ido acrecentando hasta la actualidad.

Por otra parte, es importante definir algunos conceptos importantes asociados al diseño industrial:

Producto tangible: Es un conjunto de productos que presentan atributos y características físicas que denotan su calidad y estilo, dentro de ellos también están presentes el envase, el embalaje y la marca.

Proceso de producción: Es un conjunto de operaciones a través de las que los factores se transforman en productos que pueden ser bienes físicos o servicios [8]. Estos procesos son los encargados de dar valor a los conceptos de diseño propuestos y a los materiales que se utilizarán en el producto.

Innovación: La Unesco (1977) indica que la innovación involucra el empleo de los resultados de la investigación fundamental y aplicada en la introducción de nuevas aplicaciones o en la mejora de aplicaciones ya existentes.

Estrategia: H. Koontz en su libro, Estrategia control y operación (1991), menciona que, las estrategias son el conjunto de acciones que llevan consigo responsabilidades, tareas y recursos para lograr una misión. Es decir, el conjunto de objetivos, a través de los que se busca cumplir con los propósitos de una organización siguiendo un rumbo previamente planificado. Por otro lado, el Diseño estratégico, se encarga de analizar las capacidades del diseño en el sector estratégico, proyectual y operativo de la organización industrial, para generar esquemas de interdisciplinariedad y trabajo en equipo, así como control sobre los procesos y en general las acciones necesarias para llevar a cabo las estrategias, a fin de facilitar el análisis y toma de decisiones sobre los proyectos por implementar, el control sobre las actividades y el tiempo necesario para cumplir con los deseos y demandas de los usuarios [9]. 


\subsection{Metodologías del diseño industrial}

Tradicionalmente la fabricación y el diseño estuvieron unidos en la creación de objetos, no obstante, es a partir del auge industrial, donde la figura del diseñador toma gran importancia, nace la necesidad de integrar al diseño una metodología que sea capaz de describir al artefacto a diseñar de acuerdo a los requerimientos demandados por clientes y productores, y resolver problemas complejos de diseño. Esto es corroborado por [10] quienes sostienen que, el objetivo principal del diseño industrial es satisfacer las necesidades de los usuarios a través de la creación de productos y servicios. De esto se destaca que la principal relación que resuelve el diseño industrial son los aspectos entre el usuario y el producto antes que las relaciones internas del producto, por lo tanto, las actividades que incluyen los aspectos técnicos están relacionadas con la ingeniería antes que con el diseño.

Según [11], está metodología se basa en el estudio de los principios, prácticas y procedimientos de diseño en un sentido amplio, con el objetivo de establecer estructuras apropiadas para el proceso de diseño, que incluyen procesos de análisis y síntesis a fin de dar solución a problemas de diseño.

Los métodos del diseño históricamente pueden clasificarse en métodos artesanales o intuitivos y en métodos contemporáneos, donde los primeros resultan insuficientes en muchos casos, dada la gran complejidad que requieren un elevado número de proyectos industriales. Los métodos contemporáneos son procedimientos elementales y con una finalidad parcial que cumplen determinadas misiones en el proceso de diseño[12].

Sin embargo, según Níquel Cross, existe otra clasificación, según las etapas de diseño en las que se interviene, mientras que para [13], los métodos del diseño están clasificados desde la perspectiva del cliente, del diseñador y de lacomunidad.

Actualmente existe un sin número de métodos y metodologías del diseño, las cuales encajan en las clasificaciones anteriores, algunas pueden estar inmersas o requerir de otras metodologías para su aplicación. Existen metodologías que desarrollan el diseño en todas sus fases, desde la concepción de la idea, hasta la disposición final del producto diseñado. Mientras que algunas metodologías o métodos se usan únicamente como herramientas para el análisis de un factor específico del diseño. Los métodos de interés en el presente artículo son los siguientes:

- Metodología para el desarrollo de productos del INTI.

- Metodología del ecodiseño.

- QFD (Despliegue de la función de calidad).

- Design Thinking.

- Economía Circular.

A continuación, se definen los aspectos más importantes de estás metodologías:

\subsection{Metodología para el desarrollo de productos del INTI}

Esta metodología propuesta por el Instituto Nacional de Tecnología Industrial (INTI) de la República de Argentina, se enfoca en el desarrollo total de un producto que sea capaz de ser producido industrialmente, en cada una de las etapas siguientes: Definición estratégica, Diseño de concepto, Diseño en detalle, Verificación y testeo, Producción, Mercado, y, Disposición final [14].

\subsection{Metodología del Ecodiseño}

El Ecodiseño se define como: Proceso de diseño que considera los impactos medioambientales en todas las etapas del proceso de diseño y desarrollo de productos, para lograr productos que generen el mínimo impacto medioambiental posible a lo largo de todo su ciclo de vida.

Los pasos para seguir para el desarrollo de un proyecto de ecodiseño varían de acuerdo a los actores, pero en términos generales, el ecodiseño conlleva los siguientes pasos:

Paso 1.- Organización del proyecto y selección del producto

Paso 2.- La estrategia del Ecodiseño

Paso 3.- ¿Cómo mejorar el producto? Obtención de ideas

Paso 4.- Concreción del producto: pliego de condiciones y desarrollo Metodología de Ecodiseño 
Paso 5.- Desarrollo a detalle del concepto seleccionado

Paso 6.- Plan de Acción a futuro en el producto y en la empresa

Paso 7.- Comunicación exterior de un proyectode Ecodiseño.

\subsection{QFD (Despliegue de la función de calidad)}

Es un sistema que busca focalizar el diseño de los productos y servicios para dar respuesta a las necesidades de los clientes. Lo que implica traducir la voz del cliente en requerimientos de diseño y parámetros técnicos de producción, estos requerimientos están analizados en función de las características de la competencia, los requerimientos importantes de los clientes, la dificultad organizacional de cada propuesta de mejora y las correlaciones existentes entre aquella propuesta. Además, el QFD es una herramienta dinámica que permite controlar los requerimientos de los clientes y evaluar los posibles cambios que puedan darse en el tiempo.

\subsection{Design Thinking}

Su nombre lo indica, esta metodología se enfoca en la solución de problemas, con ideas innovadoras exclusivamente enfocadas en el diseño. La aplicación de esta metodología genera grandes beneficios para las organizaciones en el diseño de soluciones, sobre todo en la comercialización. Es una metodología centrada en las personas, en sus necesidades y en la manera de satisfacer esas necesidades de una manera que sea tecnológicamente factible.

En general el "Design Thinking" empieza empatizando con las personas que están relacionadas con el producto o servicio de interés, para definir el problema en base a la información obtenida, el siguiente paso es idear soluciones que posteriormente se convertirán en modelos, los cuales se pondrán a prueba antes de su definitiva implementación.

\subsection{Economía Circular}

La economía circular es un modelo de gestión que busca el desarrollo económico de las organizaciones, basado en un enfoque ambiental y social, además, crea oportunidades de mejora o de nuevos negocios.

Se inspira en los sistemas naturales y la forma en que estos sistemas administran los flujos de materiales y energía. Los principios de la economía circular son:

Desechos = Alimento: este principio identifica a los desechos de un sistema o proceso como la entrada (alimento) para un nuevo sistema o proceso.

La diversidad crea resiliencia: este principio hace énfasis en la capacidad de los sistemas naturales de ser fuertes mientras más elementos diferentes estan relacionados con el sistema, es decir mientras más elementos están inmersos activamente en los sistemas o procesos, el sistema será menos propenso a tener fallas o a desaparecer.

Uso de energías renovables: Aquí se intenta que la energía necesaria para el funcionamiento del sistema o proceso sea energía renovable la cual además de generar beneficios ambientales, permita el ahorro de dinero para las organizaciones.

Pensar en sistemas: La economía circular trata de pensar en el beneficio y desarrollo de todos los elementos que están involucrados en los sistemas (organizaciones, proveedores, personas, gobierno, naturaleza, clientes, etc.), y en la importancia de que el sistema funcione adecuadamente para un modelo exitoso de negocios.

\subsection{Alcance y áreas de aplicación del diseño in- dustrial}

Actualmente, el diseño industrial es conocido por un mayor número de personas, sin embargo, su noción se limita a breves rasgos de lo que implica esta actividad y los campos de desarrollo de un diseñador industrial, lo que se debe a que ciertas compañías diseñan, desarrollan y producen productos propios, haciendo entrever que no se necesita de esta actividad en la organización. El campo laboral del diseñador industrial se ha ampliado, permitiéndole relacionarse con el usuario por sus nuevos diseños y creativas invenciones que tienen 
como objetivo principal la producción de manera industrial.

El diseñador industrial encuentra su principal campo de acción en la industria de la transformación y la maquila, además cuenta con un amplio conocimiento de innovación y producción por lo que puede desarrollarse en empresas públicas, privadas y organismos descentralizados o ejercer en forma independiente mediante el lanzamiento de cualquier tipo de producto, servicio o emprendimiento [15].

Un diseñador industrial tiene la capacidad de transformar la materia prima en productos altamente funcionales y estéticos, este puede usar todos los recursos existentes a su favor con el fin de satisfacer las necesidades de un público objetivo. Además, tiene la capacidad de dirigir un grupo de trabajo interdisciplinario que puede estar formado por técnicos, ingenieros, diseñadores, psicólogos, entre otros, que favorecen el desarrollo de productos capaces de satisfacer sus necesidades físicas, síquicas, económicas y emocionales. Por otra parte, el diseñador industrial tiene una amplia gama de posibilidades para desarrollarse en el campo laboral tales como [16]:

- Planificación y desarrollo de productos

- Participación en la investigación y testeo de productos

- Participación en la estandarización de líneas de producción.

- Evaluación de patentes y marcas

- Colaboración en la vigorización de la pequeña y mediana industria

- Asesoría corporativa

- Colaboración en la enseñanza teórico-práctico del diseño

\section{- Rediseño de productos existentes}

En conclusión, el diseño industrial es una rama de diseño dedicada a la representación y creación de productos, estéticos, funcionales y ergonómicos aptos de ser producidos industrialmente, lo que da lugar a condiciones y comportamientos a los que debe adaptarse el diseñador y el tejido productivo a fin de adelantarse a las nuevas exigencias del mercado.

\subsection{Gestión del diseño}

La Gestión del Diseño es una disciplina empresarial que emplea la gestión de proyectos, el diseño, la estrategia y las técnicas de la cadena de suministro para intervenir en el proceso creativo, apoyar la cultura de creatividad y construir una estructura y una organización para el diseño [17].

Es necesario hablar de la gestión del diseño al igual que la gestión de cualquier otra actividad dentro de la empresa ya que el producto es el centro de funcionamiento de la empresa y es su razón de ser. La gestión del diseño dentro de la empresa se debe dar desde la concepción del diseño a fin de asignar los recursos suficientes a las actividades que se tienen programadas a lo largo del proceso creativo y productivo.

El departamento de diseño mantiene una estrecha relación entre los departamentos de marketing y producción con la finalidad generar productos que satisfagan las necesidades de los consumidores sin olvidar los requisitos de costes y producción, como se observa en la Figura 1. Una concepción de diseño ordenada asegura que un producto funcione de la manera que fue pensada y al mismo tiempo que el usuario lo interprete con el concepto que fue creada.

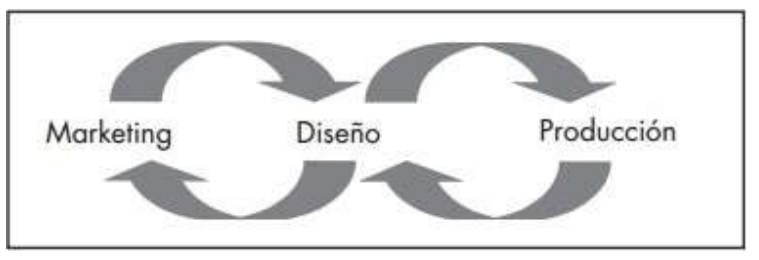

Figura 1. Relación del diseño con el marketing y la producción [1].

De igual manera, es importante conocer que un buen diseño ocasionalmente significa mayores ganancias para la empresa, ya que permite un precio superior al dar una mayor percepción de calidad al usuario. Además, la gestión del diseño le da coherencia a la empresa, ya que, el diseño de producto, diseño gráfico y diseño del entorno crean la imagen de la empresa y los comunican a través del producto, lo que ubica al diseño como eje fundamental dentro de la empresa. 


\subsection{Gestión estratégica empresarial}

La gestión estratégica empresarial tiene una estrecha relación con la administración tradicional, pero toma más importancia a los siguientes elementos:

- La visión de la organización

- La actuación prospectiva de la organización

- Organización y definición de la dirección de la empresa

- Enfoque hacia el talento humano de la empresa considerado el pilar más valioso de la organización

- Enfoque en las metas a largo plazo

Por otra parte, se basa en las cinco fuerzas competitivas de la gestión empresarial: la amenaza de nuevos entrantes, la amenaza de productos o servicios sustitutivos, el poder negociador de los proveedores, el poder negociador de los compradores y la rivalidad entre los competidores existentes, tomando en cuenta que el diseño debe estar en lo más alto de la estrategia de la empresa.

Estas fuerzas obedecen a cada sector de la industria que forma parte de su estructura, es por ello que una empresa puede generar ventaja competitiva al desarrollar características superiores a sus competidores. Una de estas es la variación de su mercado, otra es diferenciando sus costes, obteniendo una diferenciación visible por los consumidores. Razón por la cual las estrategias genéricas serían: liderazgo en costes, diferenciación y enfoque en un segmento.

\subsection{Modelos de estrategia empresarial}

La ventaja competitiva radica en ver a una empresa como un todo, ya sea en el diseño, producción, mercadotecnia, entrega y apoyo de sus productos.

En una serie de libros que se publicaron entre los años 1980 y 1990, por el economista e ingeniero eléctrico Michael Porter, reconocido por temas de estrategias de empresas, menciona que las ventajas competitivas vienen de actividades empresariales internas, sugiriendo que los factores primordiales que determinan la rentabilidad de un negocio [18] y son:

- Las cinco fuerzas que moldean la estructura de una industria, las mismas que resultan crucia- les desde un punto de vista de la formulación estratégica, de esta manera siempre estará relacionada la empresa con su medio ambiente. El objetivo que presenta es encontrar una posición en el sector en donde crezca como industria teniendo a su favor las fuerzas competitivas, sin duda alguna la innovación es una poderosa herramienta mediante la cual accede a decisiones estratégicas [19].

- Cadena de valor, sirve para evaluar el de posicionamiento una empresa.

En la Figura 2, se muestra la cadena de valor.

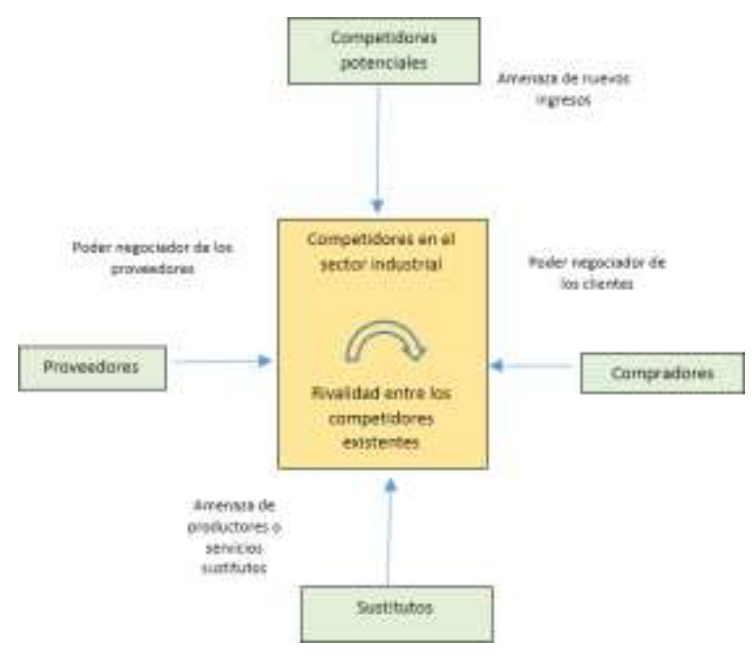

Figura 2. Cadena de Valor [19].

La estrategia del mejor producto se sustenta en la forma clásica de competencia que declara que hay sólo dos formas de ganar: bajos costos o diferenciación en diseño de un producto o servicio. El problema es que la diferenciación es rara vez una fuente de ventaja sostenible, dado que una vez que la estrategia es revelada y se hace conocida, la tecnología a menudo permite una rápida imitación que neutraliza la ventaja competitiva buscada [18] [19].

La cadena de valor es interesante cuando se considera una filosofía holística del diseño. El valor del diseño ha sido reconocido como factor importante dentro de la práctica operaciones industriales y desarrollo de productos. Por ello, se exponen modelos de estrategias empresariales que ayudan con esta problemática. 


\subsection{El modelo Delta}

Uno de los aspectos más destacados del "Delta Model" deducido por Arnoldo C. Hax es que brinda asesoramiento sobre cómo elegir el posicionamiento estratégico de su negocio y brindarle las herramientas analíticas de cómo alcanzarlo. Esta es una característica que no está disponible en cualquier otro modelo de negocio.

Sin embargo, como se comienza a crear una estrategia basada en la comprensión de las necesidades individuales de los clientes. Sin duda, el objetivo estratégico fundamental es establecer una relación con el cliente, es decir, atraer, satisfacer y conservar al cliente [20], el modelo Delta ofrece tres opciones diferentes para alcanzar ese objetivo que pueden aplicarse de preferencia con un cliente a la vez:

- En la opción de mejor producto, el cliente se acerca por la superioridad de su oferta de precio - debido a su infraestructura de bajo costo - o por algún otro aspecto relacionado con la funcionalidad del producto, marca o apariencia que lo diferencie de las ofertas de la competencia.

- En la opción de soluciones totales para el cliente, el cliente se siente atraído, debido a que la empresa le está ofreciendo algo más allá del producto en sí, que implica transferir conocimiento y servicios que satisfacen las necesidades apremiantes que tiene el cliente.

- En la opción captura del sistema, la empresa ha alcanzado un dominio tan fuerte en el mercado que el cliente no tiene mejor opción que elegir dicho producto [20].

\subsection{EI Balanced Scorecard}

Según Mario Vogel, “el BSC ayuda a balancear de una forma integrada y estratégica, el progreso actual y suministra la dirección futura de la empresa, para ayudar a convertir la visión en acción por medio de un conjunto coherente de indicadores, agrupados en 4 diferentes perspectivas, a través de las cuales se puede ver el negocio en su totalidad" [19]. Este modelo se observa en la Figura 3.

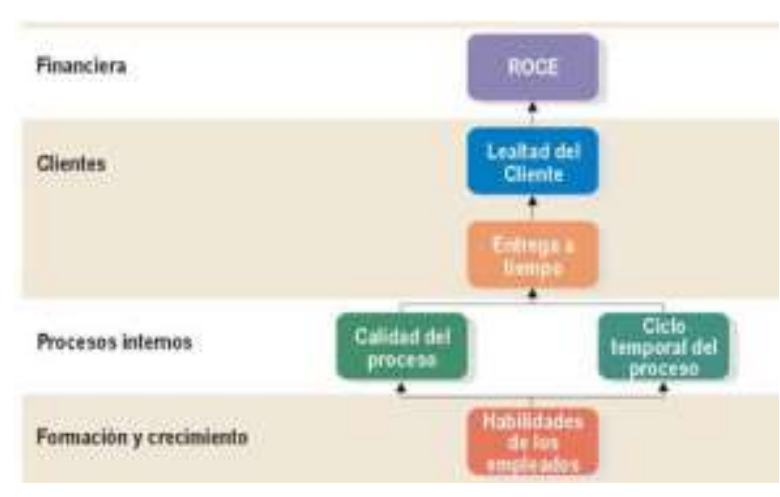

Figura 3. Modelo Balanced Scorecard [19].

Es conveniente hacer notar, que el determinante crítico para el éxito de una estrategia de innovación es la integración organizacional con otros elementos externos.

\subsection{Gestión estratégica y el perfeccionamien- to empresarial}

Consiste en el manejo del contexto de cambio, con el objetivo de alcanzar una estrategia que permita mejorar la efectividad a través de transformaciones individuales, grupales y empresariales a fin de aportar en el perfeccionamiento empresarial (Alhama, Francisco, \& Rafael, 2001). El cambio organizacional exige cambio individual, y constituye la manera más segura de llevar a cabo las transformaciones organizacionales, no obstante, es la más difícil y la menosempleada.

Tiene implicaciones directas para todos, desde la preparación y la elaboración del diagnóstico, hasta la instrumentación de la estrategia y la evaluación del avance del proceso. En este sentido, es necesario plantear y definir las necesidades de cambios y transformaciones para evitar repetir el mismo modelo. El error y la reiteración del error debe conducir al cambio y no a seguir en lo mismo y con los mismos procedimientos.

El proceso de perfeccionamiento de una empresa es ante todo un proceso originado y sostenido desde dentro, en el cual el macro entorno como sistema más amplio con sus variables económicas, político legales, ambientales, socioculturales y tecnológicas, junto con las exigencias propias de su sector de negocios deben jugar el rol de impulsores del cambio con regulaciones y especificaciones 
indispensables, pero no prescribir detalladamente en términos funcionales los procedimientos, medios ni enfoques para hacerlo. Cuando se procede de esta forma, se está reemplazando al sujeto de la transformación, quien queda como mero ejecutor de un cambio diseñado desde fuera del sistema organizacional [21].

La empresa debe desarrollar capacidades propias de perfeccionamiento, siendo su subsistema gerencial el encargado de explorar, diseñar e implementar las vías para hacerlo, a fin de asegurar su sostenibilidad interna y el cumplimiento de su responsabilidad en las dimensiones económica, sociopolítica y ambiental, bajo exigencias críticas mínimas que el entorno ha de establecer.

\subsection{Innovación empresarial}

Al incrementar la importancia de la innovación como impulsora de cambios económicos y sociales, se dedica cada vez más atención a su naturaleza, el papel que desempeña y sus determinantes. Además, la innovación es un concepto extenso que comprende una amplia gama de actividades y procesos tales como: mercados, actividades empresariales, redes y competencia, pero también las habilidades y organizaciones, la creatividad y la transferencia de conocimientos.

Por otra parte, existen tres canales que posibilitan la innovación y el rendimiento de la productividad:

I. La decisión de las empresas de invertir en la innovación;

II. El conocimiento de la función de la producción, en la que esta inversión, además de otros insumos, produce la innovación;

III. La función del rendimiento de la producción, en la que la innovación, junto con otros insumos, se relaciona con la productividad laboral.

Para una correcta creación y transferencia de conocimiento e innovación es necesario disponer de personal cualificado para ello. Por tanto, el capital humano será uno de los pilares básicos a considerar. Por otro lado, aunque relacionado con lo anterior, resulta relevante disponer de una cultura innovadora adecuada que permita la absorción y transferencia de la innovación, para lo que las TIC constituyen una de las principales herramientas
[21] [22]. Además, el espíritu empresarial existente entre la población de un país determinará la creación de empresas y por tanto la demografía empresarial, que influye sobre el total de innovaciones realizadas.

En este punto resulta crucial el acceso de las empresas a la financiación, así como los beneficios fiscales aplicados a éstas para fomentar la innovación. Otros factores con influencia sobre la demografía empresarial y por tanto sobre el nivel de innovación de un país, son la existencia de mercados competitivos y la flexibilidad de la economía y administración de este. De igual manera, el grado de cooperación intra-empresarial, intra-sectorial y entre centros de generación de conocimiento y empresas, propician una mayor transferencia y difusión del conocimiento, que resulta vital para la innovación al permitir el reparto de costes y riesgos. Por último, el sistema de innovación empresarial debería adecuarse al desarrollo sostenible del medio ambiente [23].

\section{Resultados y Discusión}

El diseño industrial constituye un instrumento estratégico, en el sentido que proporciona que los productos y servicios sean diferentes a través del proceso de innovación en los productos de una empresa. En el primer caso, el producto es la actividad clave en una empresa industrial que por medio del diseño de productos se planifica y conceptualiza el mismo. En este proceso se dota al producto del significado que va a tener para el usuario o consumidor, incluyendo las funciones, el uso, la forma y construcción.

Es importante mencionar que no todas las empresas visualizan estos beneficios, más bien, consideran que el diseño industrial como estrategia empresarial genera costos no productivos. Otros factores que han promovido o causado esta situación de apatía de estas empresas son la falta de conocimiento acerca del diseño industrial y todas las contribuciones que vienen con ello, también el desconocimiento acerca de las funciones por cada una de las partes que están inmersas en el proceso de diseño industrial; es decir, empresa y diseñador.

Una empresa al integrar el diseño industrial optimiza los recursos de esta, ya que, mejora los recursos físicos de la organización; ejemplos claros 
son los productos y envases. Por otro lado, mejora los aspectos de organización y procesos de trabajo; por ejemplo, en los edificios mediante el diseño de interiores con un adecuado reparto de espacios (Layout), inciden en la comodidad y buena comunicación tanto de usuarios como trabajadores, y evita los tiempos muertos, lo que finalmente potencializa y eleva el valor de la marca; lo que incide en la comunicación dentro y fuera de la empresa.

En la actualidad se evidencia que una empresa integra el diseño industrial al fabricar productos que no solo satisfacen las necesidades de los consumidores, sino que también, se preocupan y promueven la calidad del medio ambiente y la sostenibilidad de los recursos, llegando a consolidarse exitosos. Otros aspectos en donde se palpa la integración del diseño industrial en la empresa constituyen el valor de la producción, que permite por una parte racionalizar el proceso productivo, y por otra, contar con un análisis adecuado que reduce el número de componentes, las fases del proceso de producción y la división de operaciones productivas, consiguiendo el máximo aprovechamiento de la materia prima. Asimismo, al implementarse la filosofía de economía circular, el diseño industrial, tiene la capacidad de desarrollar nuevos productos empleando tecnología existente y aprovechando los recursos existentes a fin de optimizar la producción en sus distintas etapas.

Es indispensable mencionar que el diseño industrial facilita el comercio de productos, al optimizar y diferencir la información de la empresa y del producto; otro ejemplo que permite evidenciar la integración del diseño industrial con la gestión estratégica empresarial es el Modelo de Lean Manufacturing, que aumenta la calidad y los valores formales de los productos, gracias al valor agregado con el que se logra cada producto para diferenciarse de la competencia, al presentar ventajas funcionales, estéticas y culturales que de una u otra manera generan preferencias en los usuarios.

En el contexto de la empresa, el diseño industrial es una herramienta de gestión enfocada en aumentar la competitividad a través de la inserción de productos nuevos e innovadores, que además de satisfacer las necesidades detectadas en los consumidores, establece la diferencia con una adecuada imagen que responde a las cualidades y características implícitas en la marca y producto que se ofre- ce en el mercado. Para evidenciar esta conexión, se muestra el triángulo estratégico entre la empresa, producto, mercado y diseño industrial, que constituye un instrumento de gestión idóneo, a través de su actuación en el producto y la empresa, para posteriormente interconectar sus relaciones con un mercado determinado posibilitando el mayor grado de competitividad de la empresa.

En la Figura 4, se nuestra la relación empresa, mercado, producto que permite la competitividad en una empresa.

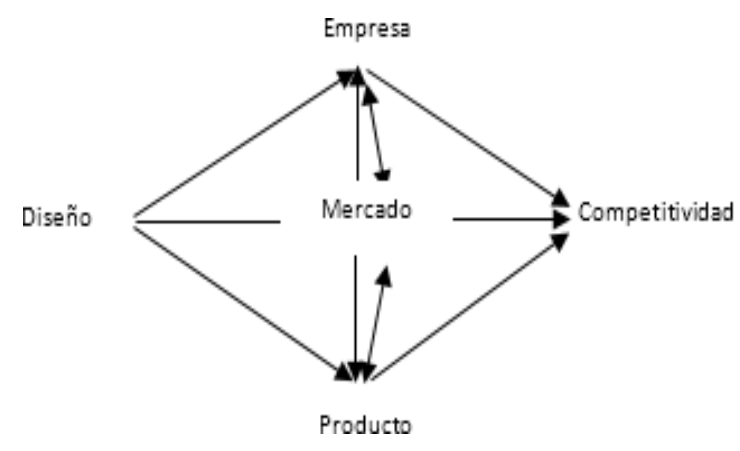

Figura 4. El diseño industrial actúa sobre la relación Empresa - producto - mercado potencializando la competitividad de la empresa [1].

La importancia de la incorporación del diseño en la empresa se ha hecho evidente a partir de los años cincuenta, cuando se ha visto la necesidad de estimular la venta a través de la manipulación del mercado por medio de la diferenciación del producto y la publicidad, aspectos que influyen en la decisión de compra, sin importar el tamaño de la empresa (Lecuona, 2006). A partir de ello, catedráticos, profesionales y empresarios en Europa y Estados Unidos, han indagado en el término y han buscado dar una adecuada integración a los procesos de diseño dentro de la organización. Sin embargo, esta disciplina toma protagonismo en los años noventa, cuando las empresas incorporan al diseño como factor estratégico, que permite involucrar las capacidades proyectuales y creativas, como recurso de innovación y competitividad (Manrique López, 2016). La Tabla 1 resume las múltiples ventajas que proporcionan cada una de las metodologías estudiadas, ya que se convierten en herramientas de gestión estratégica en las empresas, que ayudan a conseguir resultados para colocarlas en un nivel más competitivo. 
Tabla 1: Ventajas de las metodologías estudiadas [9].

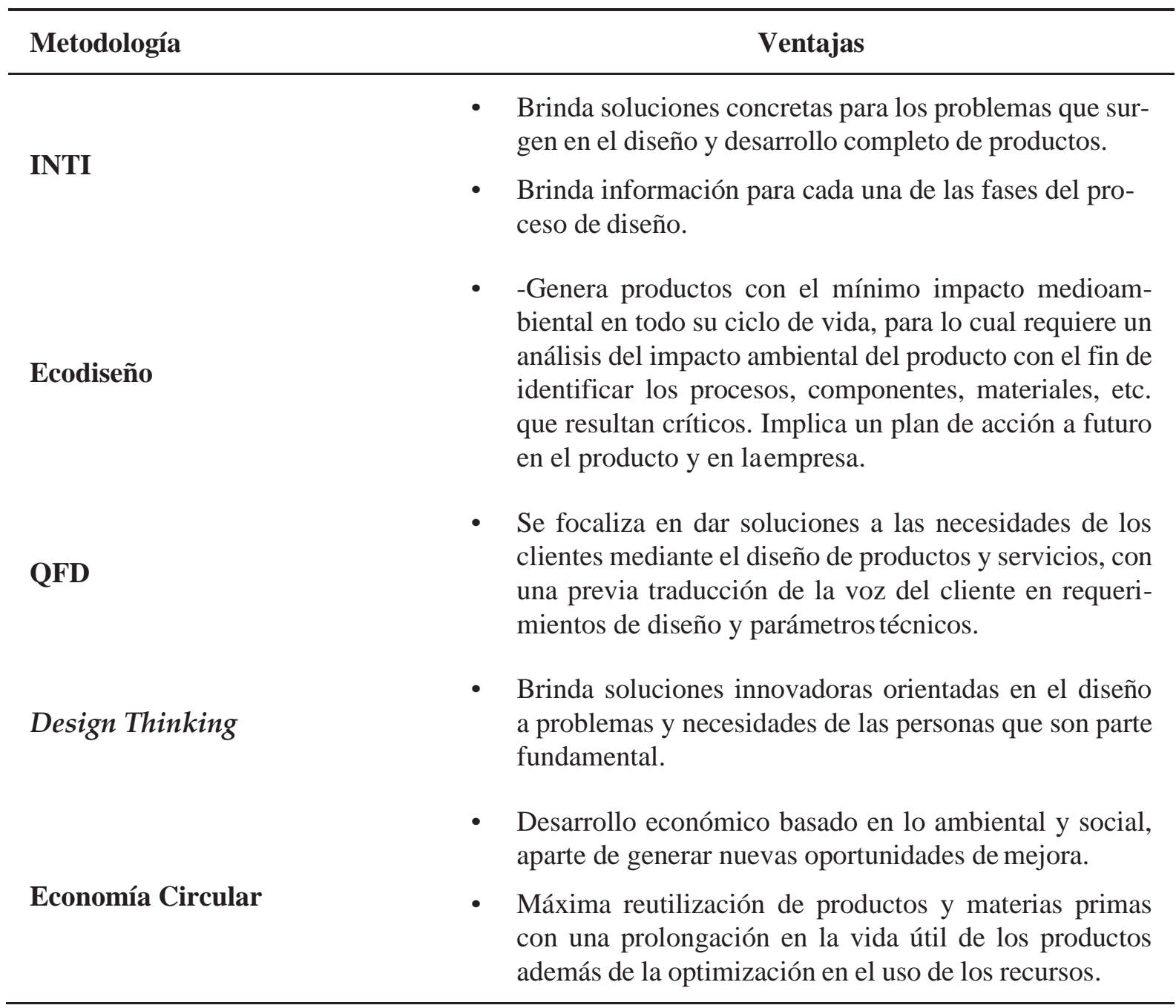

Dichas metodologías permiten definir los problemas y necesidades de los clientes (que constituyen parte fundamental de la investigación), para posteriormente convertirlos en oportunidades que ayudan a encontrar soluciones innovadoras y creativas, traducidas en productos y servicios ofertados por las empresas. En tanto que, al hablar de ecodiseño y economía circular, además de satisfacer las necesidades, su objetivo radica en el factor ambiental, ya que buscan minimizar el impacto medioambiental y optimizar los recursos, que sin duda favorecen a las empresas que buscan un mejor desarrollo económico con un mínimo empleo de recursos y perjuicios medioambientales.

Cabe mencionar que la Gestión del Ciclo de Vida de los Productos (Product Management Lifecycle) al aplicarse de forma correcta genera beneficios de sostenibilidad dentro de la economía circular [24]; al emplear esta metodología se obtienen mejores resultados que al aplicar los Sistemas de Servicios de Productos (PSS) como estrategia de los negocios que estan encaminados hacia una economía cirular. Cabe mencionar que este artículo evidencia que se obtiene una eficacia en los recursos y ahorro de costo a través de prácticas donde se aplique el ciclo de vida, incluyendo temas de economía circular desde el inicio de la vida de la oferta de PSS,de esta manera se mejora el desempeño ambiental y económico de un ente empresarial.

Se ha empleado el Modelo Estratégico Delta, realizando ajustes adecuados para mejorar las ventajas competitivas de una empresa en relación con 
otras. En la Figura 5, se observa el modelo planteado con todos los aspectos considerados con el fin de aumentar la competitividad que es clave en una empresa.

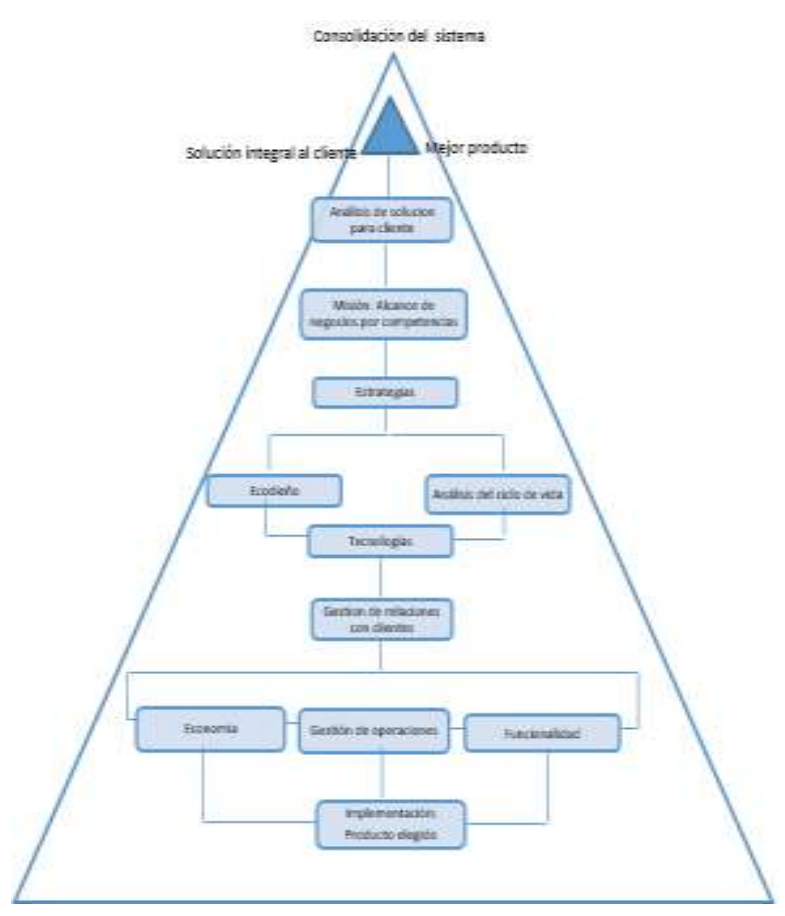

Figura 5. Modelo Delta de estrategia empresarial

\section{Conclusiones}

La integración del diseño industrial como gestión estratégica de las empresas constituye un modelo de cómo competirá la empresa, sus objetivos y las políticas necesarias para alcanzar sus objetivos a largo plazo. En fin, genera una rentabilidad a nivel económico con la optimización de recursos y procesos; además de la rentabilidad social promoviendo el cuidado ambiental a través de su ética empresarial, acompañada de la calidad de servicios con una buena atención y comunicación.

Las opciones estratégicas y competencias hacen que el modelo Delta sea una herramienta útil para que guíe su pensamiento estratégico. Es decir: la segmentación con el cliente y proposición de valor para el cliente y las competencias existentes y deseadas de una entidad empresarial.

El diseño no es sólo una herramienta que puede utilizarse para diferenciar productos y generar una visión prospectiva del sector empresarial. El diseño es también una función dentro de la estructura de la empresa que modifica los procesos y la gestión de la innovación. Al vincular el diseño con la ventaja competitiva, proporciona un modelo exploratorio que se puede utilizar de una manera prescriptiva. Cada gerente será capaz de localizar su estrategia de gestión:

- Diseño como posicionamiento diferenciador

- Diseño como posicionamiento coordinador

- Diseño como posicionamiento transformador

Por lo tanto, cada empresa puede optar por dar un valor estratégico al diseño y utilizar las variables identificadas en el estudio para hacer efectivo este proceso en su sistema de evaluación y desempeño.

En función de los objetivos y metas de cada una de las empresas, se elige la metodología más adecuada con la finalidad de generar un distintivo que les permita posicionarse en el mercado. No obstante, la decisión no se limita solo a una de ellas, ya que puede conjugarse más de una, para generar ventajas competitivas dentro de la organización.

\section{Referencias}

[1] Fundación COTEC, Diseño e innovación. La gestión del diseño en la empresa, Madrid: Gráficas Arias Montano, S. A., 2008.

[2] I. Buil, E. Martínez y T. Montaner, «Inportancia del diseño industrial en la gestión estratégica de la empresa,» UniversiaBusiness Review, pp. 52-67, 2005.

[3] B. Borja de Mozota, «Design and competitive edge: A model for design management excellence in European SMEs,» Design Management Journal, pp. 88-103, 2003.

[4] K. Clark y T. Fujimoto, Product Development Performance: Strategy, Organization, and Management in the World Auto Industry, Boston: Harvard Business School Press, 1991.

[5] F. Giraldo y E. Orozco, «Métodos deductivo e inductivo. Obtenido,» 11 Marzo 2011. [En línea]. Available: https://proyectogrado.wordpress.com/2011/03/11/ metodos-deductivo-e-inductivo/. 
[6] D. Becker, Color Trends and Selection for Product Design, Oxford: Elsevier Inc., 2016.

[7] B. Hernández, «Bauhaus la escuela que unió arte y técnica,» Jurio 2004. [En línea]. Available: http://www.tecnicaindustrial.es/tifrontal/a-1676- bauhaus-escuela-unio-arte-tecnica.aspx.

[8] F. D’Alessio Ipinza, Administración y dirección de la producción: enfoque estratégico de calidad, Bogotá: Pearson Educación Ltda, 2002.

[9] M. d. R. Rivera Sanclemelte, La evolución de las estrategias de marketing en el entorno digital: implicaciones jurídicas (Te-

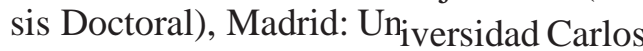
III de Madrid, 2015.

[10] M. Abdullah y R. Marshall, «A research overview of industrial de sign framework for modular product design, of the Design, pp. 95-104, 2010.

[11] N. Cross, Metodos de Diseño, Estrategias para el diseño de producps, MéxicoD. F.: Editorial Limusa, 2002.

[12] J. C. Jones, Design Meth ds, New Jersey: John Wiley \& Sons, Inc., 1992.

[13] S. Liu y I. M. Boyle, «Engineering design: perspectives, challenges and recent advances,» Journal of Engineering Design, pp. 7-19, 2009.
[15] L. E. Macías Martin y F. A. Bribiescas Silva, «Las competencias del diseño industrial en el ambito manufacturero en Ciudad Juarez.,» Nóesis, pp. 17-41, 2011.

[16] G. Rodríguez, Manual de diseño industrial, México: Ediciones G. Gili, S.A., 1983.

[17] W. Anthony, Study Guide for Human Resources Management, Cram101, 2013.

[18] M. E. Porter, Estrategia competitiva, México: CECSA, 1982.

[19] M. Aguilar Fernandez, «Los modelos mas populares de Estrategia empresarial,» UPIICSA XV, p. 6, 2007.

[20] A. C. Hax y D. L. Wilde, El modelo delta. Un nuevo marco estratégico, Dublin: Se-

[21] H. Blanco Rosales, «Hacia el perfeccionamiento empresarial: un enfoque gerencial,» CEEC, UH, La Habana, 2011.

[22] OCDE, «Innovacion en las empresas una perspectiva microeconomica,» OCDE,

París, 2012.

[23] N. Zozaya, «La innovacion empresarial en España,» Ministerio de Industria, Turismo y Comercio, Madrid, 2005.

[24] T. T. Sousa-Zomer, L. Magalhães, E. Zancul y P. A. Cauchick-Miguel, «Lifecycle Management of Product-service Systems: A Preliminary Investigation of a White 
L. 1 desarrollo de productos, " Boletín Infor-

mativo $\mathrm{N}^{\mathrm{o}}$ 141, Buenos A ires, 2009. 